\title{
TRATAMENTO CIRÚRGICO DO DIVERTÍCULO DE ZENKER: DIVERTICULOPEXIA VERSUS DIVERTICULECTOMIA
}

\section{Surgical treatment of Zenker diverticulum: diverticulopexy versus diverticulectomy}

\author{
Nelson Adami ANDREOLLO, Luiz Roberto LOPES, Nelson Ary BRANDALISE, \\ Marcelo Amade CAMARGO, João de Souza COELHO-NETO
}

ABCDDV/568

\begin{abstract}
Andreollo NA, Lopes LR, Brandalise NA, Camargo MA, Coelho-Neto JS. Tratamento cirúrgico do divertículo de Zenker: diverticulopexia versus diverticulectomia. ABCD Arq Bras Cir Dig 2007;20(4):245-9.

RESUMO - Racional - Divertículo faringoesofágico, conhecido como de Zenker, é herniação adquirida na mucosa faríngea através de um defeito muscular entre as fibras oblíquas do músculo constritor inferior da faringe e as transversas do músculo cricofaríngeo. Objetivo - estudo retrospectivo, não randomizado, compararando os resultados da diverticulopexia e diverticulectomia, ambas associadas à miotomia do músculo cricofaríngeo, no tratamento do divertículo de Zenker. Métodos - Quarenta pacientes foram submetidos a tratamento cirúrgico entre 1989 e 2003 , dos quais 38 (95\%) foram seguidos. Vinte e oito eram do sexo masculino (70\%) e 12 femininos, com média de idade de 62,5 anos (21 a 85 anos). Vinte e quatro pacientes $(60 \%)$ foram submetidos à diverticulopexia ou elevação, e 16 à diverticulectomia ou ressecção, através de cervicotomia esquerda, com miotomia do músculo cricofaríngeo. Resultados - Resultados excelentes (Visick I), foram verificados em 84,6\% dos pacientes submetidos à diverticulectomia e $66,6 \%$ dos pacientes submetidos à diverticulopexia. Na análise global de todos os casos estudados, 27 (11 ressecções e 16 elevações - 72,9\%) apresentaram Visick I; 8 (2 ressecções e 6 elevações - 21,6\%) apresentaram Visick II; e 2 (elevações - 5,4\%) apresentaram Visick III. Não foi registrado nenhum caso na classificação de Visick IV. A incidência de complicações pós-operatórias registrada nos dois grupos foi semelhante $(\mathrm{P}>0,05)$. Foi verificado a presença de neoplasia maligna em um caso submetido a ressecção (2,5\%). Conclusão - A diverticulopexia com miotomia do músculo cricofaríngeo é mais indicada em doentes geriátricos, pequenos divertículos e doentes com risco operatório elevado. A diverticulectomia é boa opção em grandes divertículos e doentes jovens, para prevenir o risco de transformação maligna. Esta casuística mostrou melhores resultados com a diverticulectomia em comparação com a diverticulopexia.
\end{abstract}

DESCRITORES - Divertículo. Esôfago. Zenker. Miotomia.

\section{INTRODUÇÃO}

Divertículo faringoesofágico, descrito inicialmente por Ludlow (1769) $)^{19}$, ou divertículo de pulsão, por Zenker $(1877)^{26}$, é herniação adquirida na mucosa faríngea através de um defeito muscular entre as fibras oblíquas do músculo constritor inferior da faringe e as transversas do músculo cricofaríngeo. Nesta localização, há área triangular bem delimitada, denominada de triângulo de Killian, na qual as fibras musculares estão rarefeitas, deixando exposta a mucosa da hipofaringe. A mucosa herniando através desta área vai formar o divertículo de Zenker (DZ) de variados tamanhos ${ }^{8}$.

A ocorrência do DZ é muito variável, sendo mais freqüentemente encontrado no norte da Europa. Não é raro na América do Norte e Austrália, mas muito incomum no Japão e Indonésia. Não há relatos sobre sua freqüência na América do Sul e Brasil, contudo é considerado como de ocorrência pouco freqüente. A sua ocorrência entre

Trabalho realizado na Disciplina de Moléstias do Aparelho Digestivo, Departamento de Cirurgia e Gastrocentro, Faculdade de Ciências Médicas da Universidade Estadual de Campinas - UNICAMP - Campinas - SP

Endereço para correspondência: Nelson Adami Andreollo, e-mail: nandreollo@hotmail.com os doentes com disfagia é de 1 a $3 \%$, mais comum na faixa etária entre 60 e 90 anos, e predominante no sexo masculino $(2: 1)^{5}$.

Muitas teorias foram lançadas ao longo dos anos sobre a sua etiopatogenia, porém, nenhuma delas tem sido totalmente aceita, apesar de estudos detalhados com manometria e cinerradiografia. A maioria dos autores concorda que a seu aparecimento é secundário à obstrução funcional e aumento da pressão intraluminal ao nível do músculo cricofaríngeo, ou seja decorrente de incoordenação entre as contrações da faringe e o relaxamento do esfíncter esofágico superior. Caracteriza-se por ter evolução progressiva ao longo dos anos ${ }^{4,5,24}$.

Os sintomas mais freqüentes incluem disfagia referida na região cervical, regurgitação, halitose, tosse à ingestão alimentar e aspiração pulmonar. Ocasionalmente os pacientes poderão apresentar emagrecimento importante, chegando à desnutrição grave e com pneumonias de repetição. O diagnóstico é estabelecido mediante história clínica minuciosa, radiografias contrastadas da faringe e esôfago, endoscopia digestiva alta, e eventualmente utilizando a manometria e a cinerradiografia ${ }^{2,20}$. Entre as complicações é citada a sua perfuração, considerada muito 
rara de ocorrer ${ }^{3}$.

O tratamento é fundamentalmente cirúrgico, com diverticulectomia ou diverticulopexia, seguido de miotomia do músculo cricofaríngeo, embora nos últimos anos, muitos autores advogam o tratamento endoscópico ${ }^{2,10,17,22}$.

O objetivo deste estudo retrospectivo, não randomizado, é comparar os resultados da diverticulopexia e diverticulectomia, ambas associadas à esofagomiotomia, no tratamento do divertículo de Zenker, através da revisão da experiência ao longo de 14 anos no Hospital das Clínicas da UNICAMP.

\section{MÉTODOS}

Quarenta doentes submetidos a tratamento cirúrgico de divertículo faringoesofágico, entre 1989 e 2003, foram estudados, dos quais $38(95 \%)$ tiveram seguimento pósoperatório. Vinte e oito eram do sexo masculino $(70 \%)$ e 12 do feminino, com média de idade de 62,5 anos (21 a 85 anos).

O diagnóstico foi estabelecido mediante os sintomas e radiografias contrastadas (esofagograma) em todos os casos. A endoscopia digestiva foi possível de ser realizada em 29 doentes (72,5\%) e a eletromanometria em 14 (35\%).

A queixa principal foi disfagia em 29 doentes $(72,5 \%)$, regurgitação em 8 doentes (20\%), e em 1 doente $(2,5 \%)$ a tosse frequente. Associados à queixa principal, o emagrecimento importante foi referido em 10 casos $(25 \%)$ e a regurgitação em 4 casos (10\%). Dois doentes apresentavam pirose, um apresentava odinofagia e outro abaulamento cervical. O tempo de história variou de 4 meses a 40 anos, sendo que 28 doentes $(70 \%)$ relatavam sintomas de 6 meses a 3 anos.

Vinte e quatro doentes $(60 \%)$ foram submetidos à diverticulopexia ou elevação, e dezesseis (40\%) foram submetidos à diverticulectomia ou ressecção, seguido de miotomia do músculo cricofaríngeo, por meio de cervicotomia esquerda.

\section{RESULTADOS}

Os divertículos tinham cerca de 1 a $10 \mathrm{~cm}$ de extensão (média de 4,5 cm) com colo de 0,5 a 6,5 cm (média de $2,5 \mathrm{~cm}$ ). A abordagem, em todos os casos, foi através de cervicotomia esquerda longitudinal, na borda interna do músculo esternocleidomastóideo, dissecando-se todos os planos e estruturas cervicais até o isolamento completo do divertículo e de seu óstio na região póstero-inferior da faringe. A diverticulopexia foi realizada em 24 pacientes (60\%) e a diverticulectomia em 16 pacientes $(40 \%)$.

A miotomia realizada consistiu em seccionar toda a musculatura do músculo cricofaríngeo e do esôfago cervical, na extensão aproximada de $3 \mathrm{~cm}$, expondo toda a mucosa. Após a diverticulectomia, o fechamento do óstio diverticular foi com sutura contínua, utilizando-se fio absorvível de acido poliglicólico número 000 , sendo que esta sutura foi posteriormente protegida com a musculatura da faringe. Em todos os casos os divertículos ressecados foram enviados para estudo anatomopatológico.

A opção pela diverticulopexia foi nos doentes portadores de divertículos de pequeno e médio tamanho, sendo que foi realizada mediante elevação e retificação do mesmo, seguido de fixação na musculatura da faringe, com fio inabsorvível de prolene 0000, evitando-se passar os pontos na sua luz, ou lacerá-lo. Foram realizados como procedimentos associados ao tratamento do divertículo, a hiatoplastia e gastrofundoplicatura a Nissen modificada em três doentes, a colecistectomia em um doente e a vagotomia troncular e gastroenteroanastomose em um doente, todos através de cirurgia video-laparoscópica.

$\mathrm{O}$ estudo anatomopatológico dos divertículos ressecados revelou processo inflamatório crônico em todos os casos. Em um paciente de 79 anos, com três anos de história, tabagista e etilista foi encontrado espessamento da parede com ulceração na mucosa, sendo demonstrado a presença de tumor maligno neuro-endócrino $(2,5 \%)$. Este doente faleceu cerca de 2 meses após a operação, decorrente de acidente vascular cerebral.

As complicações pós-operatórias consistiram de três fístulas cervicais ( 2 após diverticulopexia e 1 após diverticulectomia), sendo que, duas delas associadas a abscesso local entre o terceiro e o quarto dia de pós-operatório, com resolução em 8 a 20 dias. Seis doentes (15\%) referiram parestesia no local da incisão e outros seis apresentaram disfonia no pós-operatório, com melhora no pós-operatório tardio. Um caso apresentou hematoma na ferida operatória, com resolução ambulatorial completa em 30 dias e um outro teve infecção do trato urinário (Tabela 1). Além disso, um doente de 61 anos com hipertensão arterial e que foi submetido a diverticulopexia evoluiu para óbito $(2,5 \%)$ no primeiro dia de pós-operatório em decorrência de infarto agudo do miocárdio e acidente vascular cerebral.

TABELA 1 - Complicaçõoes pós-operatórias comparadas entre os dois grupos

\begin{tabular}{lccc}
\hline \multirow{2}{*}{ Resultados } & \multicolumn{3}{c}{ Pacientes } \\
\cline { 2 - 4 } & Div. & Divp. & $\%$ \\
\hline Parestesia na ferida operatória & $2(12.5 \%)$ & $4(16.6 \%)$ & - \\
Paralisia da corda vocal/disfonia & $3(18.7 \%)$ & $3(12.5 \%)$ & 3,2 \\
Fístula & $1(6.2 \%)$ & $2(8.3 \%)$ & 1,8 \\
Com infecção da ferida & 0 & $2(8.3 \%)$ & 3,0 \\
Recorrência & 0 & $1(4,1 \%)$ & 1,2 \\
Mortalidade & $1(6.2 \%)$ & 0 & 1,2 \\
Neoplasia & 0 & $1(4,1 \%)$ & 1,2 \\
\hline
\end{tabular}

Div: diverticulectomia; Divp: diverticulopexia.

O período de hospitalização pós-operatória variou de dois a 12 dias, com média de 4,7 dias por doente, sendo que 21 doentes permaneceram entre 2 e 4 dias internados $(52,5 \%)$.

A melhora na disfagia foi notória e referida por todos os doentes logo após a operação. Um doente perdeu totalmente o seguimento, sendo que o tempo de seguimento pós-operatório tardio de 37 doentes foi de dois meses a 11 
anos (média de 14 meses). Empregando a classificação de sintomas de Visick, 27 doentes (11 ressecções e 16 elevações) apresentaram Visick I ( $73 \%$ ), 8 doentes ( 2 ressecções e 6 elevações) apresentaram Visick II (21,6\%); e 2 doentes (2 elevações) apresentaram Visick III $(5,4 \%)$. Nenhum doente foi classificado em Visick IV (Tabela 2).

\section{TABELA 2 -}

\begin{tabular}{lcccc}
\hline \multirow{2}{*}{ Resultados } & \multicolumn{4}{c}{ Pacientes } \\
\cline { 2 - 5 } & Visick & Div. & Divp. & \% \\
\hline Excelente & I & $11(84.6 \%)$ & $16(66.6 \%)$ & 82 \\
Bom & II & $2(15.4 \%)$ & $6(25 \%)$ & 11 \\
Regular & III & 0 & $2(8.4 \%)$ & 3 \\
Ruim & IV & - & - & 4 \\
\hline
\end{tabular}

Div: diverticulectomia; Divp: diverticulopexia..

Na avaliação final, $84,6 \%$ dos pacientes submetidos à diverticulectomia e $66,6 \%$ dos pacientes submetidos à diverticulopexia foram classificados como excelentes (Visick I), com desaparecimento total dos sintomas disfágicos.

\section{DISCUSSÃO}

A ocorrência do DZ é pouco frequente no nosso meio, de tal forma que poucos serviços têm número grande de doentes, suficientes para dar experiência satisfatória no seu manuseio e tratamento.

O tratamento cirúrgico do $\mathrm{DZ}$ é fundamentalmente baseado na sua etiopatogenia, de tal modo que a maioria dos autores têm preconizado já há anos a diverticulectomia seguido de miotomia do esfincter cricofaríngeo ${ }^{10,11,12}$. No entanto, Aggerholm \& Illum ${ }^{1}$ mostram os resultados do tratamento de 110 pacientes realizando a diverticulectomia apenas, verificando $16 \%$ de recidivas do divertículo, valor este considerado elevado.

Gullane el al. ${ }^{11}$ compararam pela primeira vez os resultados de três procedimentos: da diverticulectomia seguido por miotomia, da cricofaringomiotomia somente, e da cricofaringomiotomia seguido por diverticulopexia, encontrando resultados muito semelhantes.

Laccourreye et al. ${ }^{15}$ compararam os resultados entre dois grupos: 14 doentes submetidos a diverticulectomia e 29 a diverticulopexia, todos seguidos de miotomia do cricofaríngeo, verificando que todos os doentes beneficiaram-se igualmente. Konowitz \& Biller ${ }^{14}$, no entanto, recomendam que a diverticulopexia deve ser reservada para pacientes de alto risco.

Loui \& Zuckerbraun ${ }^{18}$ também preocupados com as complicações da diverticulectomia em pacientes muito debilitados ou muito idosos, propõem inicialmente a realização da diverticulectomia seguido da esofagostomia cervical, e posteriormente o fechamento tardio da esofagostomia.

Witterick et al. ${ }^{25}$ realizaram a diverticulectomia e miotomia do cricofaríngeo em 18 pacientes com DZ e fizeram estudos radiológicos contrastados de controle cerca de 30 meses após, constatando recidiva de pequenos divertículos em 8 deles (44\%), que mantinham-se assintomáticos.

Ruiz-Tovar et al. ${ }^{23}$ relatam sua experiência no tratamento cirúrgico do divertículo em 27 doentes, em período de 20 anos, em que $74 \%$ dos casos foram submetidos a diverticulectomia e miotomia do músculo cricofaríngeo. As complicações somaram $21 \%$ e a taxa de recidiva foi de $4 \%$.

Leporrier et al. ${ }^{16}$ comparam os resultados de dois grupos de doentes igualmente divididos, submetidos respectivamente a diverticulectomia seguido de miotomia (19 doentes) e diverticulopexia (19 doentes) no período de 10 anos. Referem que não ocorreu mortalidade e o índice de complicações foi pequeno, sendo um caso com fístula cervical, três com pneumonias, três com disfonia transitória e um hematoma. O resultado foi considerado excelente em 36 casos e bom em 3. Concluem que a diverticulopexia é preferível em doentes geriátricos e a diverticulectomia preferencialmente deve ser empregada em grandes divertículos (maiores que $6 \mathrm{~cm}$ ) ou em doentes jovens pelo risco de malignização.

O tratamento endoscópico do DZ também encontra muitos adeptos e exige muita experiência para realizá-lo. Foi primeiro descrito na literatura por Mosher em 191721, o qual dividiu o septo entre o divertículo e o esôfago sob controle endoscópico. Porém, foram Dohlman \& Mattsson em $1960^{\circ}$ que desenvolveram instrumentos rígidos especiais para secção do referido septo, incluindo uma pinça para coagulação, evitando hemorragia local.

Van Overbeek ${ }^{24}$ relatam os resultados do tratamento endoscópico em 545 doentes ao longo de 30 anos, obtendo melhora muito satisfatória da disfagia em $91 \%$ deles, com taxa de complicações muito baixa.

Ishioka et al..$^{13}$, entre nós, relatam sua experiência utilizando fibroendoscópios para realizar a secção do septo em 42 doentes com DZ, obtendo bons resultados, com 7,1\% de recidiva da disfagia.

Bonavina et al. ${ }^{6}$ analisam os resultados tardios de 5 a 10 anos do tratamento de 297 doentes, comparando tratamento endoscópico (181 casos) com a diverticulectomia seguida de miotomia (116 casos). Referem que $92 \%$ dos doentes submetidos a tratamento endoscópico e $94 \%$ dos submetidos a operação aberta ficaram livres dos sintomas disfágicos, concluindo que ambas as técnicas apresentam bons resultados. Enfatizam que divertículos menores que 3 $\mathrm{cm}$ são contraindicação para o tratamento endoscópico.

A melhora dos sintomas e desaparecimento da disfagia (Visick I e II) ocorreu em 94,6\% dos casos desta casuística. Foi registrado apenas um óbito $(2,5 \%)$. Na avaliação final, $84,6 \%$ dos pacientes submetidos à diverticulectomia e $66,6 \%$ dos submetidos à diverticulopexia foram classificados como excelentes (Visick I), com desaparecimento total dos sintomas disfágicos.

Os pacientes submetidos a diverticulectomia necessitam ser mantidos em jejum durante cerca de 72 horas, antes de iniciar alimentação via oral, prolongando o tempo de hospitalização, enquanto que os que são submetidos a diver- 
ticulopexia podem ser realimentados mais precocemente, diminuindo a permanência hospitalar. A diverticulopexia teoricamente evita a esofagotomia durante a diverticulectomia, portanto, complicações como mediastinite, fistula cervical e estenose poderão ser evitadas. Na presente casuística a diverticulopexia foi empregada em divertículos de pequeno e médio tamanho.

Assim, a justificativa dos autores deste trabalho que preferem a diverticulopexia ao invés da diverticulectomia é quanto às complicações, menos frequentes na primeira, podendo ser empregada em doentes com risco cirúrgico elevado e nos mais idosos. Todavia, deve ser lembrada ainda possibilidade, embora muito rara, da ocorrência de neoplasias malignas no DZ, incidência por volta de $0,5 \%$. Brücher et al. ${ }^{7}$ em recente revisão também relatam a ocorrência é muito rara e fazem referência a um caso em que não foi possível o tratamento cirúrgico devido à condição clinica do doente. Na presente casuística foi encontrado um caso ainda mais raro de tumor neuroendócrino associado $(2,5 \%)$.

Concluindo, os resultados deste estudo mostram que tanto a diverticulectomia como a diverticulopexia podem ser utilizadas, pois beneficiam os doentes. A revisão da literatura mostra que é indispensável a realização da miotomia do esfincter cricofaríngeo, extendendo-a ao esôfago cervical, perfazendo cerca de $3 \mathrm{~cm}$ de mucosa exposta. Afastar a possiblidade de neoplasia associada deve ser uma das preocupações do cirurgião. A ressecção do divertículo previne a transformação maligna e pode ser uma forma de diagnosticar a mesma, tendo em vista o risco real do carcinoma "in situ" nos divertículos tratados com elevação ou não. O tratamento endoscópico é praticado e preconizado por número pequeno de especialistas. Portanto, vários métodos podem ser utilizados no tratamento do DZ. E, mesmo tratando-se de doença pouco frequente, é importante conhecer seus sinais e sintomas e diagnosticá-la logo no início, evitando complicações.

\section{CONCLUSÃO}

O tratamento cirúrgico do divertículo de Zenker através de cervicotomia esquerda e miotomia, seja ele associado à elevação ou ressecção do divertículo, é procedimento seguro e efetivo, com alto índice de sucesso em longo prazo e baixas taxas de morbidade e mortalidade. A diverticulopexia é mais indicada em doentes geriátricos, pequenos divertículos e doentes com risco operatório elevado. A diverticulectomia é boa opção em grandes divertículos e doentes jovens, para prevenir o risco de transformação maligna. A incidência de complicações nos pacientes submetidos à diverticulectomia não é maior que a apresentada nos submetidos à diverticulopexia e a melhora dos sintomas se mostrou mais efetiva no primeiro grupo deste estudo.

Andreollo NA, Lopes LR, Brandalise NA, Camargo MA, Coelho Neto JS. Surgical treatment of Zenker diverticulum: diverticulopexy versus diverticulectomy. ABCD Arq Bras Cir Dig 2007;20(4):245-9

ABSTRACT - Background - Pharyngoesophageal diverticulum, known as Zenker, is an acquired herniation in the pharyngeal mucous through a muscular defect between the oblique fibers of the constrictor muscle of the pharynx and the transverse fibers of the cricopharyngeal muscle. Aim - A non-randomized retrospective study was performed, comparing the results of the diverticulopexy and diverticulectomy, both associated to the cricopharyngeal myotomy, in the Zenker diverticulum treatment. Methods - Forty patients were submitted to surgical treatment between 1989 and 2003, of which 38 (95\%) were followed. Twenty-eight patients were males (70\%), with an average age of 62,5 years (21 to 85 years). Twenty-four patients $(60 \%)$ were submitted to diverticulopexy or elevation, and sixteen to the diverticulectomy or resection, through left cervicotomy, followed by cricopharyngeal myotomy. Results - Excellent results (Visick I) were found in 84,6\% of the patients submitted to the diverticulectomy and 66,6\% of the patients submitted to the diverticulopexy. General analysis showed that 27 cases (11 resections and 16 elevations - 72,9\%) presented Visick I; 8 cases ( 2 resections and 6 elevations - 21,6\%) presented Visick II; and 2 cases (elevations - 5,4\%) presented Visick III. No cases were registered under Visick IV classification. The incidence of postoperative complications recorded in the two groups was similar $(p>0,05)$. The presence of malignant neoplasia was verified in a case submitted to resection (2,5\%). Conclusion - The cricopharyngeal myotomy and diverticulopexy is suitable in geriatric patients, small diverticulum, and patients with operatory risk. Diverticulectomy is a good option in cases of larger diverticulum and young patients to prevent the risk of malignant transformation. This casuistic presented better results with diverticulectomy than diverticulopexy.

HEADINGS - Diverticulum. Esophagus. Zenker. Myotomy

\section{REFERÊNCIAS}

1. Aggerholm K \& Illum D - Surgical Treatment of Zenker's DiverticulumJ. Laryngol Otol 1990; 104:312-14

2. Andreollo NA, Soares Jr. C, Coelho Neto JS, Lopes LR, Brandalise NA, Leonardi LS. Tratamento cirúrgico do divertículo de Zenker. Rev Col Brás Cirurg 1998; 25(1):9-14

3. Andreollo NA, Brandalise NA, Leonardi LS, Boin IFSF. Divertículo de Zenker perfurado. J Bras Med 1985; 47(3): 107-110

4. Andreollo NA, Brandalise NA, Leonardi LS - Esfincter Esofágico Superior - Estudo Manométrico em Indivíduos Normais. Rev Col Bras Cirurg 1984; $11: 1-4$

5. Andreollo NA, Lopes LR, Brandalise NA - Distúrbios Motores do Esôfago.
In: Endoscopia Digestiva. 1a Edição. Rio de Janeiro: Sobed, Medsi, 1994. pp 58-68.

6. Bonavina L, Bona D, Abraham M, Saino G, Abate E. Long-term results of endosurgical and open surgical approach for Zenker diverticulum. World $\mathrm{J}$ Gastroenterol. 2007;13(18):2586-9.

7. Brücher BL, Sarbia M, Oestreicher E, Molls M, Burian M, Biemer E, Stein HJ. Squamous cell carcinoma and Zenker diverticulum. Dis Esophagus. 2007;20(1):75-8

8. Cook IJ, Blumbergs P, Cash K, Jamieson GG, Shearman DJ. - Structural abnormalities of the cricopharyngeus muscle in patients with pharyngeal (Zenker's) diverticulum. J Gastroenterol Hepatol 1992; 7:556-62 
9. Dohlman G \& Mattsson O. - The endoscopic operation for hypopharyngeal diverticula. A roentgencinematographic study. Arch Otolaryngol 1960; 71:74452

10. Ellis FH, Schlegel JF, Lynch VP, Payne WS - Crycopharyngeal myotomy for Pharyngoesophageal diverticulum. Ann Surg 1969; 170: 340-9

11. Gullane PJ, Willet JM Heeneman H - Zenker's Diverticulum. J. Otolayngol $1983 ; 12: 53-57$

12. Hunt PS, Connel AM, Smiley TB - The cricopharyngeal sphincter in gastric reflux. Gut 1970; 34:1590-9

13. Ishioka L, Sakai P, Maluf Filho, F - Endoscopic incision of Zenker's Diverticula. Endoscopy 1995; 27:433-437

14. Konowitz PM \& Biller HF - Diverticulopexy and Cricopharyngeal Myotomy: Treatment for the high-risk patients with a Pharyngoesophageal (Zenker's) Diverticulum. Otolaryngol Head Neck Surg 1989; 100:146-153

15. Laccourreye O, Ménard M, Cauchois R, Huart J, Jouffre, V, Brasnu, D, Laccourreye H - Esophageal Diverticulum: Diverticulopexy versus Diverticulectomy. Laryngoscope 1994; 104:889-92

16. Leporrier J, Salamé E, Gignoux M, Ségol P. Zenker's diverticulum: diverticulopexy versus diverticulectomy. Ann Chir. 2001;126(1):42-5

17. Lerut T, Van Raemdonck D, Guelinckx P, Dom R, Geboes K. - Zenker's diverticulum: is a myotomy of the crycopharyngeus useful ? How long should it be ? Hepatogastroenterology 1992; 39:127-31

18. Louie HW \& Zuckerbraun L - Staged Zenker's Diverticulectomy with Cervical Esophagostomy and Secondary Esophagostomy Closure for Treatment of Massive Diverticulum in Severely Debilitated Patients. Am Surg 1993; 59:842-845
19. Ludlow A. - A case of obstructed deglutition, from a preter natural dilatation of and bag formed in the pharynx. Med Soc Phys 1767; 3:35-101

20. Migliore M, Payne H, Jeyasingham, K - Pathophysiologic Basis for Operation on Zenker's Diverticulum. Ann Thorac Surg 1994; 57:1616-21

21. Mosher HP. - Webs and pouches of the esophagus, their diagnosis and treatment. Surg Gynecol Obst 1917; 25:175-87

22. Pinotti HW, Cecconello I, Zilberstein B, Pollara WM - Tratamento Cirúrgico do Divertículo Faringo-Esofágico. In: Pinotti HV et al (eds.): Atlas de Cirurgia do Esofago.1a Edição. São Paulo, 1983, pp 54-59.

23. Ruiz-Tovar J, Pérez de Oteyza J, Collado MV, Rojo R, García Villanueva A. 20 years experience in the management of Zenker's diverticulum in a third-level hospital. Rev Esp Enferm Dig. 2006;98(6):429-35

24. Van Overbeek JJM - Meditation on the pathogenesis of Hypopharyngeal (Zenker's) Diverticulum and a Report of Endoscopic Treatment in 545 patientsAnn Otol Rhinol Laryngol 1994; 103:178-185

25. Wittrick IJ, Gullane PJ, Yeung, E - Outcome analysis of Zenker's Diverticulectomy and Cricopharyngeal Myotomy. Head \& Neck 1995;17:382-88

26. Zenker FA \& Von Ziemssen H - Krankheiten des Oesophagus. In: Von Ziemssen H, ed. Hankbuch der Speciellen Pathologie und Therapie, vol 7 (suppl). Leipzig: FCW Vogel, 1877: 1-87

Conflito de interesse: não há Fonte financiadora: não há Recebido para publicação: 22/05/2007 Aceito para publicação:03/09/2007 